\title{
Study of the time reversal symmetry in the decay of ortho- Positronium atoms using the J-PET detector
}

\author{
Juhi Raj ${ }^{1, *}$ and Michał Silarski ${ }^{1}$ \\ On behalf of the J-PET Collaboration \\ ${ }^{1}$ Faculty of Physics, Astronomy and Applied Computer Science, Jagiellonian University, \\ S. Łojasiewicza 11, 30-348 Kraków, Poland
}

\begin{abstract}
The Jagiellonian Positron Emission Tomograph (J-PET) is a novel device based on organic scintillators being developed at Jagiellonian University in Kraków, Poland. J-PET is an axially symmetric and high acceptance scanner that can be used as a multi-purpose detector system. It is well suited to pursue tests of discrete symmetries in decays of positronium in addition to medical imaging. J-PET enables measurement of both momenta and polarization vectors of annihilation photons. The latter is a unique feature of the J-PET detector which allows study of the time reversal symmetry violation operator constructed solely from the annihilation photons momenta before and after scattering within the detector.
\end{abstract}

\section{Introduction}

Time reversal symmetry violation has not been observed in purely leptonic systems so far. The best experimental upper limits for CP and CPT (C-Charge Conjugation, P-Parity and $\mathrm{T}$-Time) symmetries violation in positronium decay are set to about $0.3 \times 10^{-3}$ [1-3]. According to the Standard Model predictions, photon-photon interaction or weak interaction can mimic the symmetry violation at the level of $10^{-9}$ and $10^{-13}$, respectively [4-7]. Thus, there is about 7 orders of magnitude difference between the present experimental upper limits and the Standard Model predictions. So far, discrete symmetries were proposed to be tested with the ortho-Positronium (o-Ps) system by determining the non-zero expectation values of the operators constructed from the spin $(\vec{S})$ of the o-Ps and the momentum vectors of the annihilation photons $\vec{k}_{1}, \vec{k}_{2}$ and $\vec{k}_{3}$, as presented in Table 1 . The observation of non-zero expectation values of these operators would imply non-invariance of these symmetries for which the given operator is odd (marked " - " in Table 1). In this article we propose the test of fundamental discrete symmetry violation in a purely leptonic system via operators involving polarization vectors of the annihilation photons, as proposed in Ref [8].

\section{Jagiellonian Positron Emission Tomograph}

Positron emission tomography (PET) is a non-invasive technique used in the diagnostics of various types of tumors at the cellular level. All commercially available PET scanners utilize relatively expensive crystal detectors for the detection of annihilation photons $[16,17]$. The

\footnotetext{
*e-mail: juhi.raj@doctoral.uj.edu.pl
} 
Table 1. Discrete symmetry odd-operators using spin orientation of the o-Ps, polarization and momentum directions of the annihilation photons.

\begin{tabular}{|l|c|c|c|l|l|}
\hline Operator & C & P & T & CP & CPT \\
\hline$\vec{S} \cdot \vec{k}_{1}$ & + & - & + & - & - \\
$\vec{S} \cdot\left(\vec{k}_{1} \times \vec{k}_{2}\right)$ & + & + & - & + & - \\
$\left(\vec{S} \cdot \vec{k}_{1}\right) \cdot\left(\vec{S} \cdot\left(\vec{k}_{1} \times \vec{k}_{2}\right)\right)$ & + & - & - & - & + \\
$\vec{\epsilon}_{1} \cdot \vec{k}_{2}$ & + & - & - & - & + \\
$\vec{S} \cdot \vec{\epsilon}_{1}$ & + & + & - & + & - \\
$\vec{S} \cdot\left(\vec{k}_{2} \times \vec{\epsilon}_{2}\right)$ & + & - & + & - & - \\
\hline
\end{tabular}

Jagiellonian Positron Emission Tomograph (J-PET) is the first PET scanner constructed using plastic scintillator strips to make the tomograph cost effective and portable $[9,10,13,26,27]$. The J-PET detector consists of 192 plastic scintillator strips (EJ-230) of dimensions $500 \times$ $19 \times 7 \mathrm{~mm}^{3}$ each, forming three concentric layers ( 48 modules at radius $425 \mathrm{~mm}, 48$ modules at radius $467.5 \mathrm{~mm}$ and 96 modules at radius $575 \mathrm{~mm}$ ), as shown in (Fig. 1)[13]. The
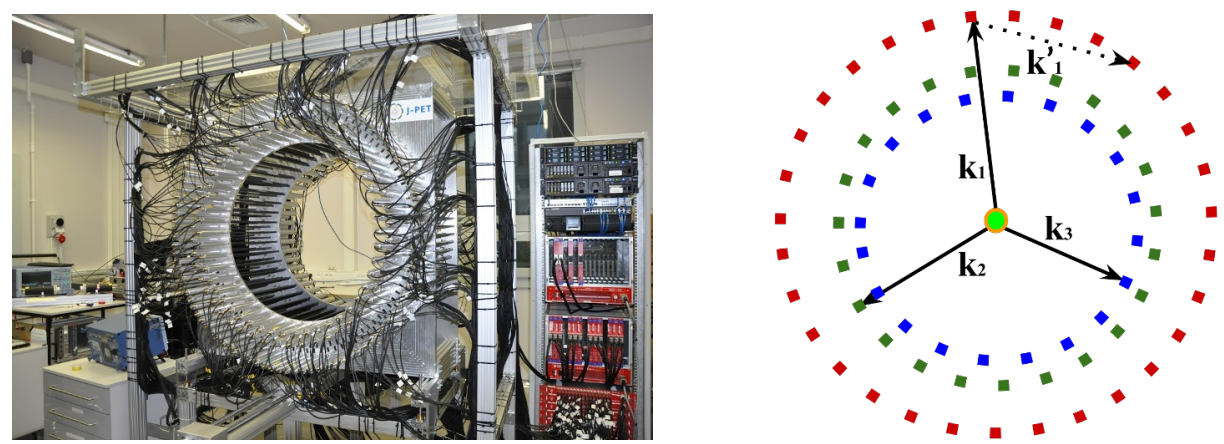

Figure 1. (Left) Front view of the J-PET detector. (Right) Schematic view of the J-PET detector with three distinguishable layers of scintillators at blue, green and red concentric cylinders. A positron source (green) is placed in the center, covered with XAD-4 porous polymer (orange). The superimposed arrows indicate the annihilation photons from ortho-Positronium decay $\left(k_{i}, k_{j}\right.$ and $\left.k_{k}\right)$, and scattered photon $\left(k_{i}^{\prime}\right)$.

J-PET detector, together with the trigger-less DAQ system constitutes an efficient photon detector with high timing properties $[13,28,29]$. One of the unique features of the J-PET detector is its ability to measure polarization of the annihilation photons. Polarization is determined as a vector product of the momenta of the photon before and after the scattering in the detector. This allows us to investigate the fundamental discrete symmetries in the purely leptonic sector [8]. In order to produce positronium atoms, a point-like ${ }^{22} \mathrm{Na}$ source is placed in the center of the detector and is surrounded with XAD-4 porous polymer [19]. The porous polymer enhances the ortho-positronium formation. For the present studies of the time reversal symmetry, we measure the expectation value of $\vec{\epsilon}_{2} \cdot \vec{k}_{1}$ operator. Thus, we are interested in events with three reconstructed gamma quanta originating from the $o P s \rightarrow 3 \gamma$ annihilation, where, one of them is scattered enabling us to determine it's polarization.

\section{Conclusion}

Positronium is an excellent laboratory enabling studies of many interesting phenomena [21] such as e.g. gravitation of antimatter [21], search for mirror photons [22, 23], studies of quantum entanglement $[24,25]$ and tests of discrete symmetries in the leptonic sector [8]. So 
far, T-violation effects were not discovered in a purely leptonic system e.g in the positronium system. The J-PET detector offers high acceptance in the measurement of the direction of polarization $(\vec{\epsilon})$ of photons simultaneously with their momentum direction $(\vec{k})$. High accuracy of time measurement together with an effective reduction of the background based on the event geometry should allow to increase the sensitivity by one order of magnitude with respect to the current experimental limits [8].

\section{Acknowledgement}

This work was supported by The Polish National Center for Research and Development through grant INNOTECH-K1/IN1/64/, 159174/NCBR/12, the Foundation for Polish Science through the MPD and TEAM/2017-4/39 programmes, the National Science Centre of Poland through grants no. 2016/21/B/ST2/01222, 2017/25/N/NZ1/00861, the Ministry for Science and Higher Education through grants no. 6673/IA/SP/2016, 7150/E338/SPUB/2017/1 and 7150/E-338/M/2017, DSC grant no. 7150/E-338/M/2018 and the Austrian Science Fund FWF-P26783.

\section{References}

[1] V. A. Kostelecky and N. Russell, Rev. of Mod. Phys. 83, 11(2011)

[2] T. Yamazaki et al., Phys. Rev. Lett. 104, 083401 (2010)

[3] P. A. Vetter and S.J. Freedman, Phys. Rev. Lett. 91, 263401 (2003)

[4] M. S. Sozzi, Discrete Symmetries and CP Violation, Oxford University Press (2008)

[5] W. Bernreyther et al., Z. Phys. C 41, 143 (1988)

[6] B. K. Arbic et al., Phys. Rev. A 37, 3189 (1988)

[7] A. Pokraka and A. Czarnecki, Phys. Rev. D96, 093002 (2017)

[8] P. Moskal et al., Acta Phys. Polon. B 47, 509 (2016)

[9] P. Moskal et al., Phys. Med. Biol. 61, 2025-2047 (2016)

[10] P. Moskal et al., Nucl. Instr. and Meth. A 764, 317 (2014)

[11] D. Kamińska et al., Eur. Phys. J. C 76, 445 (2016)

[12] A. Gajos et al., Nucl. Instr. Meth. A 819, 54 (2016)

[13] S. Niedźwiecki et al., Acta Phys. Pol. B 48, 1567 (2017)

[14] G. Korcyl et al., Acta Phys. Polon. B 47, 491 (2016)

[15] W. Krzemień et al., Acta Phys. Polon. A 127 1491-1494 (2015)

[16] S. Vandenberghe et al., EJNMMI Physics 3(1), 3 (2016)

[17] P. Slomka et al., Seminars in Nucl. Medicine, 46(1), 5 - 19 (2016)

[18] G. Korcyl et al., IEEE Transactions on Medical Imaging (2018), in print

[19] B. Jasińska et al., Acta Phys. Polon. B 47, 453 (2016)

[20] M. Pałka et al., JINST 12 P08001, (2017)

[21] D. B. Cassidy, Eur. Phys. J. D 72: 53, (2018)

[22] C. Vigo et al., arXiv:1803.05744v2 [hep-ex] (2018)

[23] S. N. Gninenko et al., Mod. Phys. Lett. A, Vol. 17, No. 26 1713-1724 (2002)

[24] B. C. Hiesmayr and P. Moskal, Scientific Reports 7: 15349 (2017)

[25] M. Nowakowski and D. B. Fierro, Acta Phys. Polon. B 48, 1955 (2017)

[26] P. Moskal et al., Nucl. Instrum. and Meth in Physics Research A 775 54-62 (2015)

[27] P. Kowalski et al., Phys. Med. Bio., arXiv:1808.00241 (2018)

[28] L. Raczyński et al., Phys. Med. Bio., 62, 5076-5097, (2017)

[29] L. Raczyński et al., Nucl. Instrum. and Meth., A 786, 105-112, (2015) 